PROF. DAVID ALAN ELLWOOD (Orcid ID : 0000-0003-4512-6443)

Article type : Mini commentary

Mini-commentary on BJOG-20-0029.R1: Prediction of stillbirth: an umbrella review of evaluation of prognostic variables

\title{
Challenges in developing prediction models for stillbirth
}

Authors: Jessica Sexton ${ }^{1,2}$, David Ellwood ${ }^{1,2}$, Vicki Flenady ${ }^{1,3}$

${ }^{1}$ NHMRC Centre of Research Excellence in Stillbirth, Mater Research Institute, Brisbane, Australia

${ }^{2}$ School of Medicine, Griffith University, Southport, Australia

${ }^{3}$ University of Queensland, Brisbane, Australia

Correspondence: David Ellwood d.ellwood@griffith.edu.au

Stillbirth is a major global public health problem, but in the absence of a robust method to predict a woman's individualized risk of stillbirth, identifying women at increased risk remains a challenge. Awareness of factors that increase risk is a necessary step in improving care through better communication and shared decision-

This article has been accepted for publication and undergone full peer review but has not been through the copyediting, typesetting, pagination and proofreading process, which may lead to differences between this version and the Version of Record. Please cite this article as doi: $\underline{10.1111 / 1471-0528.16525}$

This article is protected by copyright. All rights reserved 
making, with the goal of reducing stillbirths. Despite a high proportion of unexplained stillbirths, many women have one or more risk factors that are often unrecognized (Flenady, V. et al Lancet 2011, 377, 133140). In this umbrella systematic review, Townsend et al. have comprehensively summarized reviews of candidate predictors in regression models (BJOG $\mathrm{xxxx}$ ). Umbrella reviews are designed to enable a clear understanding of a broad topic area (Aromataris, E. et al International journal of evidence-based healthcare $\mathbf{2 0 1 5}, 13,132-40)$. A strength of this approach is the inclusion of reviews that report quantitative evidence beyond randomized controlled trials, which is where the bulk of evidence on risk factors for stillbirth exists.

An important functional distinction should be made between simple or multi-variable models and prediction models. 'Prediction model' is often misused as a blanket term in situations where predictive performance is not considered. The minimum qualification to classify a statistical model as a prediction model should be the presence of internal validation, which includes transparent reporting of model specifications and performance (Collins, G. S. et al, Annals of Internal Medicine 2015, 162, 55-63). These are criteria that can be used to assess the ability of a risk factor, with clinical and statistical association, to predict an outcome (stillbirth). Among existing prediction models, few have been statistically validated, many do not meet recommended reporting standards, and it is unknown whether they are translatable into practice.

Prediction models for stillbirth often rely on population-level information available in perinatal surveillance and hospital datasets. Due to low stillbirth prevalence, 'big data' approaches are favorable to ensure statistical powering and representation. Criticism of existing prediction models developed from routine data sources, due to poor sensitivity and specifity, appears to be misplaced. Age, parity, and prior adverse pregnancy outcomes are established risk factors for stillbirth and highlighted in this review as high-quality candidate predictors. The review findings are supported by a recent prediction model that correctly classifies stillbirth at an excellent performance level using a combination of socio-demographics, chronic conditions, and current pregnancy complications and characteristics (Malacova, E. et al, Scientific Reports 2020, 10, 5354). It seems attainable to develop a stillbirth prediction model using information currently available.

Improving the quality of prediction models with internal and external validation, transparent reporting, and consideration for clinical translation, is necessary to make them useful for detecting individualized stillbirth risk and informing clinical decision-making. One example is the argument for analytic focus on developing stillbirth prediction models for specific settings. In external validation, a model is tested in a population

This article is protected by copyright. All rights reserved 
separate from that used in model development. This is a critical step for model transportability and has a great impact on whether the model can be applied to diverse clinical settings. Overall, higher quality models developed using predictors that are both sensitive and specific are needed to reach clinically acceptable performance thresholds.

No disclosures: Completed disclosure of interest forms are available to view online as supporting information.

This article is protected by copyright. All rights reserved 